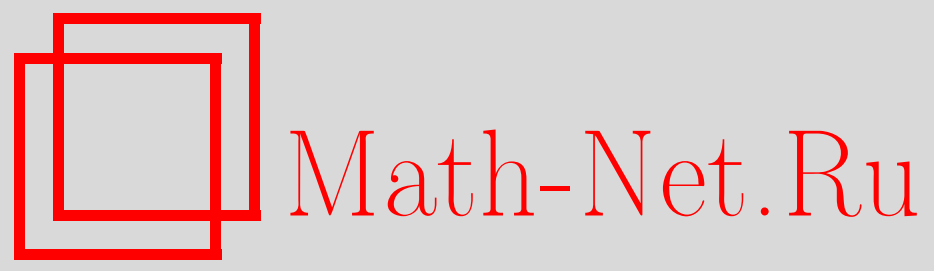

М. К. Кравцов, Неразрешимость задач векторной дискретной оптимизации в классе алгоритмов линейной свертки критериев, Дискрет. матем., 1996, том 8, выпуск 2, 89-96

DOI: https://doi.org/10.4213/dm518

Использование Общероссийского математического портала Math-Net.Ru подразумевает, что вы прочитали и согласны с пользовательским соглашением http: //www . mathnet.ru/rus/agreement

Параметры загрузки:

IP: 54.205 .225 .156

26 апреля 2023 г., 10:31:09

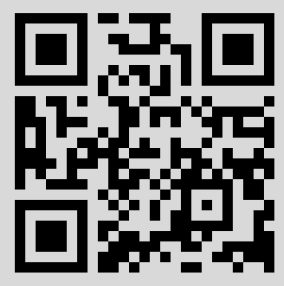


УДК 519.10

\title{
Неразрешимость задач векторной дискретной оптимизации в классе алгоритмов линейной свертки критериев
}

\author{
(c) 1996 г. M. К. Кравцов
}

\begin{abstract}
В терминах систем подмножеств описан достаточно тирокий класс задач комбинаторной векторной оптимизации, которые неразрешимы $c$ помощью классического приема линейной свертки критериев. В әтот класс, в частности, попадают хорошо известные задачи на графах (коммивояжора, о цепях между двумя вершинами и совершенных паросочетаниях, о р-медиане и покрытии графа цепями), а также разнообразные задачи булева программирования с векторной целевой функцией, представляющей собой любую комбинацию критериев вида MAXSUM, MAXMIN, MAXMAX.

Работа частично финансировалась Фондом фундаментальных исследований Республики Беларусь.
\end{abstract}

Системой подмножеств (CII), как обычно $[1,2]$, назовем пару $\tau=(E, T)$, где $E$ - конечное множество әлементов, $T$ - некоторая совокупность его непустых подмножеств $t$, называемых далее траекториями. На множестве $E$ зададим векторную весовую функцию (ВB $\Phi)$

$$
W(e)=\left(w_{1}(e), w_{2}(e), \ldots, w_{r}(e)\right),
$$

где $w_{s}(e) \in \mathbf{R}_{+} \forall s \in N_{r}=\{1,2, \ldots, r\}, \forall e \in E$, а на множестве траекторий $T-$ векторную целевую функцию (ВЩ $\Phi)$

$$
F(t)=\left(F_{1}(t), F_{2}(t), \ldots, F_{r}(t)\right), \quad F_{s}(t) \rightarrow \max _{t} \quad \forall s \in N_{r}
$$

с частными критериями трех видов:

MAXSUM:

$$
F_{s}(t)=\sum_{e \in t} w_{s}(e) \rightarrow \max _{T}
$$

MAXMIN:

$$
F_{s}(t)=\min _{e \in t} w_{s}(e) \rightarrow \max _{T}
$$


MAXMAX:

$$
F_{s}(t)=\max _{e \in t} w_{s}(e) \rightarrow \max _{T}
$$

ВЦФ $F(t)$ будем называть комбинированной, если она представляет собой любую комбинацию критериев вида MAXSUM, MAXMIN и MAXMAX, т.е. либо содержит все три вида названных критериев, либо любые два из них, либо один из них.

ВЦФ $F(t)$ определяет на $T$ паретовское множество (ПМ) $\tilde{T} \subseteq T$, состоящее из всех паретовских оптимумов: $\tilde{t} \in \tilde{T}$ тогда и только тогда, когда не существует такой траектории $t^{\prime} \in T$, что

$$
F(\tilde{t}) \neq F\left(t^{\prime}\right), \quad F(\tilde{t}) \leqslant F\left(t^{\prime}\right) .
$$

Говоря об $r$-критериальной комбинаторной задаче $\left(r\right.$-ККЗ) $Z^{r}=(E, T, W, F)$, будем подразумевать задачу нахождения ПМ $\tilde{F}$. При этом под $r$-ККЗ понимается массовая задача $[1,3]$. Наряду с массовой задачей будем использовать и понятие индивидуальная задача. Всякая индивидуальная $r$-ККЗ определяется конкретным набором $(E, T, W, F)$, где СП $\tau=(E, T)$ и все параметры ВЦФ $F(t)$, в том числе и ВВ $\Phi W(e)$, фиксированы. СП $\tau$ в әтом случае называется индивидуальной $\mathrm{C \Pi}$, а $W(e)$ индивидуальной $\mathrm{BB} \Phi$.

Очевидно, что в схему $r$-ККЗ вкладываются такие известные задачи на графах, как задачи о коммивояжере, о совершенных паросочетаниях, об остовных деревьях, о цепях между парой вершин и другие, поскольку множество $E$ можно трактовать как множество ребер графа, а траектории, соответственно, как гамильтоновы циклы, совершенные паросочетания, остовные деревья и простые цепи между выделенной парой вершин. В эту же схему вписываются и задачи булева программирования (см. [2]).

Один из важнейших подходов к нахождению паретовских оптимумов в многокритериальных задачах (любой природы, как непрерывных, так и дискретных) основан на линейной свертке критериев $[4,5]$, сущность которой заключается в скаляризации задачи, т.е. в свертывании векторного критерия в одну числовую функцию. Преимущество такого подхода состоит в возможности применения эффективного аппарата теории оптимизации, именуемого алгоритмами линейной свертки критериев (АЛСК). К сожалению, АЛСК не всегда гарантируют нахождение всех паретовских оптимумов в задачах векторной дискретной оптимизации. Это означает, что существует такая индивидуальная задача, в которой некоторый паретовский оптимум никакой линейной сверткой критериев выловить не удается. В этом случае принято говорить, что массовая задача неразрешима с помощью АЛСК [6-10].

В [6-10] доказано, что многокритериальные задачи на графах (об остовных деревьях и цепях между парой вершин, о совершенных паросочетаниях и коммивояжере) неразрешимы с помощью АЛСК, если ВЦФ состоит лишь из критериев вида MINSUM. Этот результат позже был обобщен в $[11,12]$ на случай, когда ВЦ $\Phi$ представляет собой любую комбинацию критериев вида MINSUM и MINMAX. Установлена также неразрешимость с помощью АЛСК и многокритериальных задач о р-медиане графа, о покрытии графа звездами (цепями, циклами) и разнообразных задач о назначениях с теми же критериями $[11,12]$. 
В настоящей работе исследуются $r$-ККЗ с комбинированной ВЦФ. Показано, что такие задачи при некоторых дополнительных условиях на СП неразрешимы с помощью АЛСК. Этот результат, в частности, позволяет установить неразрешимость с помощью АЛСК многокритериальных задач на графах (коммивояжера, о цепях между парой вершин и совершенных паросочетаниях, о $p$-медиане и покрытии графа цепями), а также разнообразных задач булева программирования с комбинированной ВЦФ.

Индивидуальную СП $\tau=(E, T)$ назовем особой, если существуют три попарно различные траектории $t_{1}, t_{2}, t_{3}$ и әлементы $e_{k}^{*} \in t_{k} \backslash t^{(0)}, k=1,2,3$, удовлетворяющие условиям

$$
\begin{array}{r}
\left\{t \in T: t \subseteq t\left(e_{1}^{*}, e_{2}^{*}, e_{3}^{*}\right)\right\}=\varnothing \\
\bigcup_{D \in \mathfrak{B}} t(D)=\varnothing,
\end{array}
$$

где

$$
t^{(0)}=\bigcup_{1 \leqslant k_{1}<k_{2} \leqslant 3}\left(t_{k_{1}} \cap t_{k_{2}}\right), \quad t\left(e_{1}^{*}, e_{2}^{*}, e_{3}^{*}\right)=\bigcup_{k=1}^{3}\left(t_{k} \backslash\left\{e_{k}^{*}\right\}\right),
$$

$\mathfrak{B}$ - множество всех подмножеств из $\left\{e_{1}^{*}, e_{2}^{*}, e_{3}^{*}\right\}$, состоящих не менее, чем из двух элементов,

$$
t(D)=\{t \in T: D \subseteq t\} \quad \forall D \in \mathfrak{B} .
$$

Такую тройку траекторий $t_{1}, t_{2}, t_{3}$ также будем называть особой.

Теорема 1. Для өсякой особой CП $\tau=(E, T)$ с комбинированной ВЦФ $F(t)$ существует такая индивидуальная $B B \Phi W(e)$, что для индивидуальной $r-K K 3$ $(E, T, W, F), r \geqslant 2$, выполняется условие

$$
\exists \tilde{t} \in \tilde{T}: \quad \Phi(\tilde{t}, \lambda)<\max \{\Phi(t, \lambda): t \in T\} \quad \forall \lambda \in \Lambda_{r},
$$

әде

$$
\begin{gathered}
\Phi(t, \lambda)=\sum_{s=1}^{r} \lambda_{s} F_{s}(t), \quad \lambda=\left(\lambda_{1}, \ldots, \lambda_{r}\right) \in \Lambda_{r}, \\
\Lambda_{r}=\left\{\lambda=\left(\lambda_{1}, \ldots, \lambda_{r}\right): \sum_{s=1}^{r} \lambda_{s}=1, \lambda_{s}>0 \forall s \in N_{r}\right\} .
\end{gathered}
$$

Доказательство. Сначала рассмотрим случай двух критериев $(r=2)$. Пусть $\tau=(E, T)-$ особая CП, а $t_{1}, t_{2}, t_{3}-$ особая тройка траекторий в $\tau$. Определим индивидуальную ВВФ

$$
W(e)=\left(w_{1}(e), w_{2}(e)\right)
$$

следующим образом:

$$
\begin{aligned}
w_{1}\left(e_{1}^{*}\right)=0, \quad w_{2}\left(e_{1}^{*}\right)=a, \\
w_{1}\left(e_{2}^{*}\right)=a, \quad w_{2}\left(e_{2}^{*}\right)=0, \\
w_{1}\left(e_{3}^{*}\right)=w_{2}\left(e_{3}^{*}\right)=b, \\
w_{s}(e)=\delta_{s} \quad \forall e \in t\left(e_{1}^{*}, e_{2}^{*}, e_{3}^{*}\right), \quad s=1,2, \\
w_{1}(e)=w_{2}(e)=0 \quad \forall e \in E \backslash\left(\bigcup_{k=1}^{3} t_{k}\right),
\end{aligned}
$$


где

$$
\delta_{s}= \begin{cases}0, & \text { если } F_{s}(t) \text { - критерий вида MAXSUM или MAXмAX, } \\ c, & \text { если } F_{s}(t) \text { - критерий вида MAXMIN, }\end{cases}
$$

$c>a>2 b>0$.

Тогда, учитывая соотношения (4) и (5), заключаем, что, какие бы виды критериев из (1)-(3) ни фиксировались в качестве функций $F_{1}(t)$ и $F_{2}(t)$, для всякой траектории $t \in T$ возможна лишь одна из следующих альтернатив:

(1) $F_{1}(t)=0, F_{2}(t) \leqslant a$,

(2) $F_{1}(t) \leqslant a, F_{2}(t)=0$,

(3) $F_{1}(t)=F_{2}(t)=b$.

Поэтому, принимая во внимание очевидные равенства

$$
\begin{array}{ll}
F_{1}\left(t_{1}\right)=0, & F_{2}\left(t_{1}\right)=a, \\
F_{1}\left(t_{2}\right)=a, & F_{2}\left(t_{2}\right)=0, \\
F_{1}\left(t_{3}\right)=F_{2}\left(t_{3}\right)=b,
\end{array}
$$

получим, что

$$
\begin{gathered}
\left\{t_{1}, t_{2}, t_{3}\right\} \subseteq \tilde{T}, \\
b=\Phi\left(t_{3}, \lambda\right)<\frac{a}{2} \leqslant \max \left\{\Phi\left(t_{1}, \lambda\right), \Phi\left(t_{2}, \lambda\right)\right\} \leqslant \max \left\{\Phi(t, \lambda: t \in T\} \quad \forall \lambda \in \Lambda_{2} .\right.
\end{gathered}
$$

Отсюда следует справедливость теоремы 1 в случае, когда $r=2$.

Доказательство теоремы 1 в случае $r>2$ получим, если весовые функции $w_{1}(e)$ и $w_{2}(e)$ оставим прежними, а при $s=3,4, \ldots, r$ положим

$$
\begin{array}{ll}
w_{s}(e)=w_{2}(e) & \forall e \in E \backslash t\left(e_{1}^{*}, e_{2}^{*}, e_{3}^{*}\right), \\
w_{s}(e)=\delta_{s} & \forall e \in t\left(e_{1}^{*}, e_{2}^{*}, e_{3}^{*}\right) .
\end{array}
$$

Теорема 1 доказана.

Теорема 2. Следующие $r$-критериальжые $(r \geqslant 2)$ задачи с комбинированной ВЦФ неразреиимы с помощью АЛСК:

(1) Задача о совериенных паросочетаниях на п-вершиняом әрафе при пхбом четном $n \geqslant 4$.

(2) Задача о чепях мехсду парой өериия на п-верииняом графе при любом $n \geqslant 4$.

(3) Задача похрытия п-вериияного графа чепями [13], длижы которых прияадлехсат заданному мнохсеству $\left\{h_{1}, h_{2}, \ldots, h_{p}\right\} \subseteq\{2,3, \ldots, n\}$, для любого числа $n \geqslant 4$, представимого в виде

$$
n=\sum_{i=1}^{p} h_{i} x_{i}, \quad x_{i} \in \mathbf{Z}_{+}
$$


(4) Задача о хоммивояэсере на $п$-вериинном графе при любом $n \geqslant 6$.

(5) Задача о коммивояэсере на п-вериинмом орграфе при любом $n \geqslant 4$ [13].

(6) Задача о $p$-медиане $(1 \leqslant p \leqslant n-1)$ n-вериинного графа (со взвешенными вериинами) при любом $n \geqslant 4$ [14].

(7) Прямоугольняя задача о назначениях порядка $m \times n, 3 \leqslant m \leqslant n$ [15].

(8) Задача о назначениях с $m$ категориями работ порядка $m \times n, 3 \leqslant m \leqslant n$ [15].

(9) Распределительная задача с булевыми переменными (или, в другой терминологии, обобщенная задача о назначениях) порядка $m \times n, 3 \leqslant m \leqslant n$ [16].

(10) Задача развозки [17] (или, в другой терминологии, задача о разбиении [16]) порядка $n \geqslant 4$.

Здесь ссылки указывают на источник, где имеются постановки этих задач в однокритериальном случае $(r=1)$.

Доказательство. Так как все перечисленные выше $r$-критериальные задачи можно трактовать как $r$-ККЗ (см. $[2,12])$, в силу теоремы 1 для доказательства этой теоремы достаточно построить особую тройку траекторий некоторой индивидуальной СП для каждой из задач с учетом указанных размерностей.

Прежде всего заметим, что построенные в [12] так называемые примитивные тройки траекторий для задач 1-3 и 7-9, удовлетворяют условиям (4) и (5), т.е. являются особыми.

Теперь докажем существование особой тройки траекторий для некоторых индивидуальных СП задач о коммивояжере, $p$-медиане и задачи развозки.

4. Рассмотрим $n$-вершинный $(n \geqslant 6)$ граф $G=(V, E)$, у которого множество вершин $V=N_{n}=\{1, \ldots, n\}$, а множество ребер $E=\left\{e_{i}: i \in N_{n+5}\right\}$ определено в виде следующего списка:

$$
\begin{gathered}
e_{1}=(1,2), \quad e_{2}=(1,4), \quad e_{3}=(1,3), \\
e_{4}=(2,3), \quad e_{5}=(4,3), \quad e_{6}=(2,4), \\
e_{7}=(4,5), \quad e_{8}=(3,5), \quad e_{9}=(2,5), \\
e_{i}=(i-5, i-4), \quad i=10,11, \ldots, n+4, \quad e_{n+5}=(n, 1) .
\end{gathered}
$$

Легко убедиться, что множество всех траекторий задачи о коммивояжере на этом графе состоит из следующих шести гамильтоновых циклов:

$$
\begin{aligned}
t_{1} & =\left\{e_{1}, e_{6}, e_{5}, e_{8}\right\} \cup H, \\
t_{2} & =\left\{e_{2}, e_{6}, e_{4}, e_{8}\right\} \cup H, \\
t_{3} & =\left\{e_{3}, e_{4}, e_{6}, e_{7}\right\} \cup H, \\
t_{4} & =\left\{e_{1}, e_{4}, e_{5}, e_{7}\right\} \cup H, \\
t_{5} & =\left\{e_{3}, e_{5}, e_{6}, e_{9}\right\} \cup H, \\
t_{6} & =\left\{e_{2}, e_{5}, e_{4}, e_{9}\right\} \cup H,
\end{aligned}
$$

где

$$
H=\left\{e_{i} \in E: i=10,11, \ldots, n+5\right\}
$$


Пусть $e_{1}^{*}=e_{1}, e_{2}^{*}=e_{2}, e_{3}^{*}=e_{3}$. Тогда

$$
t^{*}=t\left(e_{1}^{*}, e_{2}^{*}, e_{3}^{*}\right)=\left\{e_{4}, e_{5}, e_{6}, e_{7}, e_{8}\right\} \cup H .
$$

Поскольку подграф $G^{*}=\left(V, t^{*}\right)$ графа $G$ не содержит гамильтоновых циклов, справедливо соотношение (4). Очевидно, что при әтом будет выполняться и условие (5). Значит, тройка траекторий $t_{1}, t_{2}, t_{3}$ является особой.

5. Рассмотрим полный $n$-вершинный орграф $K_{n}$ с множеством вершин $V=$ $N_{n}$ и множеством дуг $E=N_{n} \times N_{n}$. Очевидно, что множества дуг

$$
\begin{aligned}
& t_{1}=\{(2,1),(1,3),(3,4),(4,5), \ldots,(n-1, n),(n, 2)\}, \\
& t_{2}=\{(3,1),(1,2),(2,4),(4,5), \ldots,(n-1, n),(n, 3)\}, \\
& t_{3}=\{(4,1),(1,2),(2,3),(3,5),(5,6), \ldots,(n-1, n),(n, 4)\}
\end{aligned}
$$

являются гамильтоновыми контурами орграфа $K_{n}$, т.е. траекториями задачи коммивояжера на этом орграфе.

Пусть $e_{1}^{*}=(2,1), e_{2}^{*}=(3,1), e_{3}^{*}=(4,1)$. Тогда

$$
\begin{array}{r}
t=t\left(e_{1}^{*}, e_{2}^{*}, e_{3}^{*}\right)=\{(1,2),(1,3),(2,3),(2,4),(3,4),(3,5),(4,5),(4,6), \ldots \\
(n-1, n),(n, 2),(n, 3),(n, 4)\} .
\end{array}
$$

Поскольку подграф $G^{*}=\left(V, t^{*}\right)$ орграфа $K_{n}$ не содержит гамильтоновых контуров, справедливо соотношение (4). Ясно, что при әтом будет выполняться и условие (5). Следовательно, тройка траекторий $t_{1}, t_{2}, t_{3}$ является особой.

6. Областью определения задачи о $p$-медиане графа $(1 \leqslant p \leqslant n-1)$ [14] является множество матриц

$$
\begin{aligned}
X=\left\{x=\left\|x_{i j}\right\|_{n \times n}:\right. & \sum_{i=1}^{n} x_{i j}=1 \forall j \in N_{n}, \\
& \left.\sum_{i=1}^{n} x_{i i}=p, x_{i j} \leqslant x_{i i} \forall i, j \in N_{n}, x_{i j} \in\{0,1\} \forall(i, j) \in N_{n} \times N_{n}\right\} .
\end{aligned}
$$

Таким образом, при фиксированных $n$ и $p, 1 \leqslant p \leqslant n-1$, множество $X$ определяет единственную СП $\tau=(E, T), E=N_{n} \times N_{n}$, в которой для любого $n \geqslant 4$ тройка траекторий

$$
\begin{aligned}
& t_{1}=\{(1, n)\} \cup Q, \\
& t_{2}= \begin{cases}\{(2,1),(2,2), \ldots,(2, n)\} & \text { при } p=1, \\
\{(2, n)\} \cup Q & \text { при } p \geqslant 2,\end{cases} \\
& t_{3}= \begin{cases}\{(3,1),(3,2), \ldots,(3, n)\} & \text { при } p=1, \\
\{(1,1),(3,3),(1,2),(1,4),(1,5), \ldots,(1, n-1),(3, n)\} & \text { при } p=2, \\
\{(3, n)\} \cup Q & \text { при } p \geqslant 3,\end{cases}
\end{aligned}
$$

где

$$
Q=\{(1,1),(2,2), \ldots,(p, p),(1, p+1),(1, p+2), \ldots,(1, n-1)\}
$$


удовлетворяет, как легко видеть, условиям (4), (5), если положить $e_{1}^{*}=(1, n)$, $e_{2}^{*}=(2, n), e_{3}^{*}=(3, n)$, т. е. является особой.

10. Областью определения задачи развозки порядка $n$ [17] является множество векторов

$$
X=\left\{x=\left(x_{1}, x_{2}, \ldots, x_{n}\right): \sum_{j=1}^{n} a_{i j} x_{j}=1 \forall i \in N_{m}, x_{j} \in\{0,1\} \forall j \in N_{n}\right\},
$$

где $a_{i j}$ - заданные числа $\left(a_{i j}=1\right.$, если по маршруту $j$ обслуживается $i$-й клиент, и $a_{i j}=0$ в противном случае),

$$
\sum_{i=1}^{m} a_{i j} \geqslant 1 \quad \forall j \in N_{n} .
$$

В качестве индивидуальной СП рассмотрим область определения задачи развозки порядка $n \geqslant 4$, заданную числами

$$
\begin{aligned}
& a_{i j}=1 \quad \forall(i, j) \in N_{m-l} \times N_{n-1}, \quad 1 \leqslant l \leqslant m-1, \\
& a_{i n}=1, \quad i=m-l+1, m-l+2, \ldots, m,
\end{aligned}
$$

$a_{i j}=0$ для остальных пар $(i, j)$.

Легко убедиться, что тройка траекторий $t_{1}=\{1\}, t_{2}=\{2\}, t_{3}=\{3\}$, является особой, так как в качестве $e_{1}^{*}, e_{2}^{*}$ и $e_{3}^{*}$ можно взять соответственно 1,2 и 3.

Теорема 2 доказана.

Замечапие. Утверждение 7 теоремы 2, касающееся неразрешимости квадратной задачи о назначениях порядка $n$, может быть обобщено и на $p$-индексные $(p>2)$ как аксиальные, так и планарные задачи о назначениях порядка $n \geqslant 3$ (терминологию см. в [18]).

\section{Список литературы}

1. Пападимитриу Х., Стайглиц К. Комбияаторжая оптимизачии, алгоритмы и сложсность. Мир, Москва, 1985.

2. Емеличев В. А., Кравцов М. К. О комбинаторных задачах векторной оптимизации. Дискретная математиха (1995) 7, Ne1, 3-18.

3. Гәри М., Джонсон Д. Вычислителькье машияы и трудяорешаемые задачи. Мир, Москва, 1982.

4. Михалевич В. С., Волкович В. Л. Вичислительные методы исследования и проектировакия слохскых систем. Наука, Москва, 1982.

5. Подиновский В. В., Ногин В. Д. Парето-оптимальяые решения мяогохритериальных задач. Наука, Москва, 1982.

6. Емеличев В. А., Перепелица В. А. Многокритериальные задачи об остовах графа. ДАН СССР (1988) 298, 수, 544-547. 
7. Емеличев В. А., Перепелица В. А. К вычислительной сложности многокритериальных задач. Изө. АН СССР, Техн. киберкетика (1988) №1, 78-85.

8. Емеличев В. А., Перепелица В. А. О некоторых алгоритмических проблемах многокритериальной оптимизации на графах. Журнал вичисл. матем. и матем. физики (1989) 29, N2, 171-183.

9. Emelichev V. A., Perepeliza V. A. Complexity of vector optimization problems on graphs. Optimization (1991) 22, N26, 903-918.

10. Емеличев В. А., Перепелица В. А. Сложность дискретных многокритериальных задач. Дисхреткая математиха (1994) 6, №1, 3-33.

11. Емеличев В. А., Кравцов М. К. О неразрешимости векторных задач дискретной оптимизации на системах подмножеств в классе алгоритмов линейной свертки критериев. Докл. РАН (1994) 334, Ne1, 9-11.

12. Емеличев В. А., Кравцов М. К. О задачах векторной дискретной оптимизации на системах подмножеств, неразрешимых с помощью алгоритмов линейной свертки. Журнал өычисл. матем. и матем. физики (1994) 34, №7, 1082-1094.

13. Перепелица В. А. Многокритериальные задачи теории графов. Алгоритмический подход. УМК ВО, Киев, 1989.

14. Кристофидес Н. Теория графов. Алгоритмический подход. Мир, Москва, 1978.

15. Кравцов М. К., Шерман А. Х. О решении комбинаторных задач оптимизации с минимаксными критериями. Кибержетиха (1989) 스, 71-77.

16. Ковалев М. М. Дискретная оптимизачия. БГУ им. В. И. Ленина, Минск, 1977.

17. Корбут А. А., Финкельптейн Ю. Ю. Дискретное программироваяие. Наука, Москва, 1969.

18. Емеличев В. А., Ковалев М. М., Кравцов М. К. Ммогограякихи, графы, оптимизачия. Наука, Москва, 1981.

Статья поступила 25.01.94. 